\title{
Correction to: Pre-existing cerebrovascular disease and poor outcomes of COVID-19 hospitalized patients: a meta-analysis
}

\author{
Urvish Patel $^{1} \cdot$ Preeti Malik $^{1}$ (D) Dhaivat Shah ${ }^{1} \cdot$ Achint Patel $^{1} \cdot$ Mandip Dhamoon $^{2} \cdot$ Vishal Jani $^{3}$
}

Published online: 5 September 2020

c) Springer-Verlag GmbH Germany, part of Springer Nature 2020

Correction to: Journal of Neurology

https://doi.org/10.1007/s00415-020-10141-w

The original version of this article unfortunately contained a mistake. The correct information is given below.

Third sentence of the section "Meta-analysis" should read as

Meta-analysis of all nine studies showed that COVID19 patient with pre-existing CeVD had higher odds of poor outcomes compared to better outcomes with a pooled OR of 2.67 (95\% CI 1.75-4.06; $p \leq 0.00001$ ), with minimal heterogeneity between studies $\left(I^{2}=12 \%\right.$; $\left.\mathrm{Tau}^{2}=0.05 ; \mathrm{Chi}^{2}=9.10\right)$ (Fig. 2).

The original article can be found online at https://doi.org/10.1007/ s00415-020-10141-w.

Preeti Malik

pmalik.ma@gmail.com

1 Department of Public Health, Icahn School of Medicine at Mount Sinai, 1 Gustave L. Levy Pl, New York, NY 10029, USA

2 Department of Neurology, Icahn School of Medicine at Mount Sinai, New York, NY, USA

3 Department of Neurology, Creighton University School of Medicine, Omaha, NE, USA 